\title{
TOWERS ARE UNIVERSALLY MEASURE ZERO AND ALWAYS OF FIRST CATEGORY
}

\author{
SZYMON PLEWIK
}

(Communicated by Andreas R. Blass)

\begin{abstract}
We improve a few known results about universally measure zero and always of first category sets. Our main tool is the fact that any tower with respect to a Borel relation is such a set.
\end{abstract}

\section{INTRODUCTION}

Let $\omega$ denote the set of all natural numbers, and let $\omega_{1}$ denote the first uncountable cardinal. $P(X)$ denotes the set of all subsets of $X$ and $X \times Y$ denotes the Cartesian product of $X$ and $Y$. Ordinal numbers are denoted by $k, m, n, \alpha, \beta, \lambda$, where the first three symbols are reserved for natural numbers. An inequality $\alpha<\beta$ means that the ordinal $\alpha+1$ is a subset of $\beta$. We use the definition of ordinal numbers such that $\alpha=\{\beta: \beta<\alpha\}$; in particular, $n=\{0,1,2, \ldots, n-1\}$.

The natural topology is the topology on the set $P(\omega)$ of all subsets of natural numbers for which the sets

$$
U_{X}^{n}=\{Y \subseteq \omega: Y \cap n=X \cap n\},
$$

where $X \subseteq \omega$ and $n \in \omega$, constitute a base of open sets. Let us note that sets $U_{X}^{n}$ are closed.

A family of subsets of natural numbers is a universally measure zero set if for every countably additive nonzero and finite measure which is defined on its Borel subsets, with respect to the topology inherited from the natural topology, there is a one point set with positive measure. In the literature one can find other equivalent definitions of universally measure zero sets; see Sierpiński and Szpilrajn [11], Laver [7], or Miller [9].

There are a few known constructions of uncountable universally measure zero sets; see Miller [9]. The first ones are due to Hausdorff [5] and Sierpiński and Szpilrajn [11]. Those constructions give universally measure zero sets of cardinality $\omega_{1}$. Other constructions, which produce universally measure zero sets with cardinality greater than $\omega_{1}$ in suitable models for the ZFC axioms of set theory, were given by Grzegorek [4] and Cichon [1].

Received by the editors October 7, 1991 and, in revised form, March 13, 1992.

1991 Mathematics Subject Classification. Primary 28A05.

Supported by grant KBN Nr PB/10/IM/91. 
We describe a construction of uncountable universally measure zero sets, namely, uncountable towers with respect to a Borel relation. This gives results which seem to be new. We obtain a new proof of the fact that an $\left(\omega_{1}, \omega_{1}^{*}\right)$ gap forms a universally measure zero set; compare Hausdorff [5] and Laver [7]. We obtain universally measure zero sets which can have cardinality greater than those from Grzegorek's paper [4]. We reprove Cichon's results about so-called Banach numbers; compare Cichon [1].

A family of subsets of the natural numbers is an always of first category set if every dense-in-itself subfamily of it is a first category set in itself; compare Kuratowski [6, p. 516] or Miller [9].

In Lusin [8] it was observed that a certain construction of uncountable sets produces always of first category sets; compare Kuratowski [6, p. 519]. In this note we observe that any tower with respect to a Borel relation is simultaneously a universally measure zero set and an always of first category set.

\section{THE MAIN LEMMA}

Assume that for every natural number $n$ there is given a relation $<_{n}$ on subsets of $n$. For subsets $X$ and $Y$ of the natural numbers we write $Y<X$ if for almost all $n$ there holds $Y \cap n<_{n} X \cap n$.

We define a relation $<^{n}$ on subsets of natural numbers as

$$
<^{n}=\left\{(Y, X): Y \cap n<_{n} X \cap n\right\} .
$$

A standard quantifier-counting argument, similar to $\S 1 \mathrm{C}$ of [10], gives that any relation $<^{n}$ is a closed-open set in the product $P(\omega) \times P(\omega)$. Thus, $<$ is an $F_{\sigma}$ set in $P(\omega) \times P(\omega)$.

A family $T$ of subsets of the natural numbers is a tower (compare Dordal [3]) if it is well ordered by the relation $<$, i.e., $T=\left\{T_{\alpha}: \alpha<\lambda\right\}$ and if $\alpha<\beta$, then $T_{\alpha}<T_{\beta}$ and there does not hold $T_{\beta}<T_{\alpha}$.

The following lemma needs only the assumption that a relation $<$ is a Borel subset of $P(\omega) \times P(\omega)$. The proof of it is a modification of essentially already known results; see $[10, \S 5 \mathrm{~A} .10]$. Our main improvement is that we use a suitable definition of a universally measurable zero set (this is from Sierpiński and Szpilrajn [11]).

Lemma. Each tower is a universally measure zero and an always of first category set.

Proof. Let $T=\left\{T_{\alpha}: \alpha<\lambda\right\}$ be a tower. Suppose, to the contrary, that there exists a countably additive, nonzero, and finite measure defined on Borel subsets of $T$ such that one-point sets have measure zero. We can assume that $\lambda$ is the least ordinal for which the assumptions above are fulfilled; if necessary, one can take a suitable ordinal less than $\lambda$.

Let us consider the Borel subset $E=<\bigcap(T \times T)$ of the product $T \times T$. It is measurable with respect to product measure. Each horizontal section $E \cap$ $\left(T \times\left\{T_{\beta}\right\}\right)$ is contained in

$$
\left\{T_{\alpha}: \alpha<\beta\right\} \times\left\{T_{\beta}\right\} \cup\left\{\left(T_{\beta}, T_{\beta}\right)\right\} .
$$

For $\beta<\lambda$ the sets $\left\{T_{\alpha}: \alpha<\beta\right\}$ have measure zero. Therefore, the set $E$ has measure zero because of Fubini's theorem. 
On the other hand each vertical section $E \cap\left(\left\{T_{\beta}\right\} \times T\right)$ contains

$$
\left\{T_{\beta}\right\} \times\left\{T_{\alpha}: \beta<\alpha\right\} .
$$

For $\beta<\lambda$, sets $\left\{T_{\alpha}: \beta<\alpha\right\}$ have full measure and, in particular, have positive measure. Therefore, the set $E$ has positive measure because of Fubini's theorem. We have a contradiction, which implies that $T$ has to be a universally measure zero set.

The proof that each tower is an always of first category set is similar. One has to use the Kuratowski-Ulam theorem (see Kuratowski [6, p. 249]) instead of Fubini's theorem.

\section{ApPlications}

Let us consider the following relation on subsets of the natural numbers: $X<^{*} Y$ if the set $X$ is almost contained in the set $Y$. It can be defined in the form of our schema from the previous part. Namely, one can write $X<^{n+1} Y$ if $n \in X$ implies $n \in Y$. Hausdorff [5] gave a construction of uncountable universally measure zero sets using this relation. First he constructed an $\left(\omega_{1}, \omega_{1}^{*}\right)$ gap, i.e., two families $\left\{A_{\alpha}: \alpha<\omega_{1}\right\}$ and $\left\{\beta_{\alpha}: \alpha<\omega_{1}\right\}$ of subsets of natural numbers linearly ordered in the manner

$$
A_{0}<^{*} A_{1}<^{*} \ldots<^{*} A_{\alpha}<^{*} \cdots<^{*} B_{\alpha}<{ }^{*} \cdots<<^{*} B_{1}<{ }^{*} B_{0}
$$

and such that there does not exist a set $C$ for which there hold all the inequalities $A_{\alpha}<^{*} C<^{*} B_{\alpha}$. Then he showed that any $\left(\omega_{1}, \omega_{1}^{*}\right)$ gap forms a universally measure zero set. His proof essentially uses properties of $\left(\omega_{1}, \omega_{1}^{*}\right)$ gaps; compare Laver [7]. The last result can be deduced from our theorem. Any $\left(\omega_{1}, \omega_{1}^{*}\right)$ gap is the union of countably many towers with respect to relation $<^{*}$ and its reverse (one has to use the observation that for each $X$ there are only countably many $Y$ which satisfy $\left.X<^{*} Y<^{*} X\right)$.

In Grzegorek [4] there was given a construction of universally measure zero sets of cardinality equal to the least cadinality of nonmeasurable sets; we denote it $\operatorname{non}(L)$. One can produce a relation, via our schema from the previous part, for which there exist towers of cardinality $b$, and in some model for ZFC there can exist towers of cardinality greater than $b$; see Dordal [3]. The cardinal $b$ is taken as in Fremlin [2], where it was noted that one cannot prove $b \leq \operatorname{non}(L)$ using ZFC axioms only. So our theorem gives a stronger result than Grzegorek's in some models for ZFC.

A cardinal number $\lambda$ is called a Banach number (see Chicon [1]) if there are a universally measure zero set of cardinality $\lambda$ and a nonmeasurable subset of reals of cardinality $\lambda$. In any model for ZFC with $b>\operatorname{non}(L)$ there are at least two Banach numbers, namely, $b$ and $\operatorname{non}(L)$. Thus we have reproved the result of Cichon [1]: it is consistent with ZFC that there exist many Banach numbers.

One can define a relation as needed above as follows: $X<_{*} Y$ if $X$ is finite or if for almost all $n$ there holds $x_{n}<y_{n}$, where $x_{0}<x_{1}<\cdots$ is the increasing sequence of all members of $X$ and $y_{0}<y_{1}<\cdots$ is the increasing sequence of all members of $Y$. Thus $b$ is the least cardinality of unbounded towers with respect to the relation $<_{*}$ restricted to the set of all infinite subsets of natural numbers. The relation $<_{*}$ can be obtained in the form of the schema 
from the previous part, when one puts $X<{ }^{2 n} Y$ if for every $x_{k}$ greater than $n$ there holds $x_{k}<y_{k}$ and for odd $n$ we let $X<^{n} Y$ hold for each $X$ and $Y$.

\section{REFERENCES}

1. J. Cichoń, On Banach numbers, Bull. Acad. Polon. Sci. Ser. Math. 29 (1981), 531-534.

2. D. Fremlin, Cichon's diagram, Seminaire Initiation à l'Analyse (G. Choquet, M. Rogalski, J. Saint-Raymond, eds.), no. 23, Univ. Pierre et Marie Curie, Paris, 1983/84.

3. P. S. Dordal, Towers in $[\omega]^{\omega}$ and ${ }^{\omega} \omega$, Ann. Pure Appl. Logic, vol. 45, North-Holland, Amsterdam, 1989, pp. 247-276.

4. E. Grzegorek, Solution of a problem of Banach on $\sigma$-fields without continuous measures, Bull. Acad. Polon. Sci. Ser. Math. 28 (1980), 7-10.

5. F. Hausdorff, Summen von $\aleph_{1}$ mengen, Fund. Math. 26 (1936), 241-255.

6. K. Kuratowski, Topology. I, Academic Press, New York, 1966.

7. R. Laver, On the consistency of Borel's conjecture, Acta Math. 137 (1977), 157-169.

8. N. Lusin, Sur l'existence d'un ensemble non denombrable qui est de première catégorie dans tout ensemble parfait, Fund. Math. 2 (1921), 55-157.

9. A. W. Miller, Special subsets of the real line, Handbook of Set-Theoretic Topology (K. Kunen and J. E. Vaughan, eds.), Elsevier, Amsterdam, 1984, pp. 204-233.

10. Y. N. Moschovakis, Descriptive set theory, North-Holland, Amsterdam, 1980.

11. W. Sierpiński and E. Szpilrajn, Remarque sur le problème de la mesure ${ }^{1}$, Fund. Math. 26 (1936), 256-261.

Instytut Matematyki Uniwersytetu Ślaskiego, ul. Bankowa 14, 40007 Katowice, Poland 